\title{
LAGRANGE MULTIPLIERS FOR FUNCTIONS OF INFINITELY MANY VARIABLES*
}

BY L. W. COHEN

The purpose of this note is to extend the Lagrange multiplier theorem to the case of a maximum of a function of infinitely many variables subject to an infinity of auxiliary conditions. The underlying implicit function theorems used are due to Hart. $\dagger$ The proof employs two lemmas on normal determinants and associated linear systems of equations which seem to have been overlooked. $\ddagger$ One of these incidentally renders one assumption in Hart's implicit function theorem redundant.

LEMma 1. If $\sum_{i, k}\left|a_{i k}\right|=A$ and $A_{i k}$ is the minor of $\delta_{i k}+a_{i k}$ in the determinant $\Delta=\left|\delta_{i k}+a_{i k}\right|$, then $\sum_{i, k}\left|A_{i k}\right|(i \neq k)$ converges and the $\left|A_{i i}\right|$ are bounded.

Proof. Since $\sum_{i, k}\left|a_{i k}\right|$ converges, $\prod_{k}\left(1+\sum_{i}\left|a_{i k}\right|\right)$ converges. If $p=a_{i_{1} i_{2}} a_{i_{2} i_{3}} \cdots a_{i_{n} i_{1}}$, the infinite product $\Pi(1+|p|)$ extended over all values of $p$ is dominated by the product $\prod_{k}\left(1+\sum_{i}\left|a_{i k}\right|\right)$ and converges to a value $P$. A term of $A_{i k},(i \neq k)$, has one of the forms

$$
a_{k i} T, \quad a_{k i_{1}} a_{i_{1} i_{2}} \cdots a_{i_{n} i} T,
$$

where $T$ is a product of factors $p$ and the indices are all distinct. Hence

$$
\left|A_{i k}\right| \leqq P\left\{\left|a_{k i}\right|+\sum_{n} \sum_{i_{1} \cdots i_{n}}\left|k, i_{1}, i_{2}, \cdots, i_{n} ; i\right|\right\},
$$

where $\left|k, i_{1}, i_{2}, \cdots, i_{n} ; i\right|$ is $\left|a_{k i_{1}}, a_{i_{1} i_{2}}, \cdots, a_{i_{n} i}\right|$ or zero according as the indices are distinct or not. Now

* Presented to the Society, December 27, 1933.

$\dagger$ W. L. Hart, Differential equations and implicit functions in infinitely many variables, Transactions of this Society, vol. 18 (1917), Theorems XII, XIII, VI.

‡ For the normal determinant theory, see F. Riesz, Les Systèmes d'Equations Linéaires . . , 1913. 


$$
\begin{aligned}
& \sum_{k, i} \sum_{i_{1}, \cdots, i_{n}}\left|k, i_{1}, \cdots, i_{n} ; i\right| \\
& \quad \leqq \sum_{k, i_{1}}\left|a_{k i_{1}}\right| \sum_{i_{1} i_{2}}\left|a_{i_{1} i_{2}}\right| \cdots \sum_{i_{n} i}\left|a_{i_{n} i}\right|=A^{n+1} .
\end{aligned}
$$

Let us assume $A<1$. Then

$$
\begin{aligned}
\sum_{i, k}\left|A_{i k}\right| & \leqq P\left\{\sum_{i, k}\left|a_{k i}\right|+\sum_{i, k} \sum_{n} \sum_{i_{1} \cdots i_{n}}\left|k, i_{1}, \cdots, i_{n} ; i\right|\right\} \\
& \leqq P\left\{A+\sum_{n} A^{n+1}\right\}=\frac{P A}{1-A} .
\end{aligned}
$$

It is clear that $\left|A_{i i}\right| \leqq P$. The restriction $A<1$ may be removed by use of a convergence factor.*

Lemma 2. If $\sum_{i, k}\left|a_{i k}\right|=A, \sum_{i}\left|b_{i}\right|=B$, and $\Delta \neq 0$, then the solution $x_{k}$ of $x_{i}+\sum_{k} a_{i k} x_{k}=b_{i}$ is such that $\sum_{k}\left|x_{k}\right|$ converges.

Proof. The solution is given by

$$
x_{k}=\frac{1}{\Delta} \sum_{i} A_{i k} b_{i}=\frac{1}{\Delta}\left\{\sum_{i \neq k} A_{i k} b_{i}+A_{k k} b_{k}\right\} .
$$

Then

$$
\begin{aligned}
\sum_{k}\left|x_{k}\right| & \leqq \frac{1}{|\Delta|} \sum_{k}\left\{\sum_{i \neq k}\left|A_{i k} b_{i}\right|+\left|A_{k k} b_{k}\right|\right\} \\
& \leqq \frac{B}{|\Delta|} \sum_{k} \sum_{i \neq k}\left|A_{i k}\right|+\frac{P}{|\Delta|} \sum_{k}\left|b_{k}\right|,
\end{aligned}
$$

which, with Lemma 1, proves Lemma 2.

Let $S, T$ be the sets of points $\xi$, $\eta$ with coordinates $x_{i}, y_{i}$ such that $\left|x_{i}-a_{i}\right|<r_{i}<r,\left|y_{i}-b_{i}\right|<r_{i}^{\prime}<r,(i=1,2, \cdots)$, respectively, and let $R$ be the space $(\xi, \eta)$. A function $f(\xi, \eta)$ is called completely continuous in $R$ if to every $\epsilon>0$ there is a $\delta_{\epsilon}>0$ such that $\left|f\left(\xi^{\prime}, \eta^{\prime}\right)-f\left(\xi^{\prime \prime}, \eta^{\prime \prime}\right)\right|<\epsilon$ when $\left|x_{i}^{\prime}-x_{i}^{\prime \prime}\right|<\delta_{\epsilon}$, $\left|y_{i}^{\prime}-y_{i}^{\prime \prime}\right|<\delta_{\epsilon}$, and $\left(\xi^{\prime}, \eta^{\prime}\right),\left(\xi^{\prime \prime}, \eta^{\prime \prime}\right)$ are in $R$. The complete continuity of $f(\xi), f(\eta)$ in $S, T$, respectively, is similarly defined.

IMPlicit Function TheOREM. If

(1) $\phi_{i}(\xi, \eta)$ and $\partial \phi_{i} / \partial y_{j},(i, j=1,2, \cdots)$, are completely continuous in $R$;

* See F. Riesz, loc. cit., p. 38. 
(2) $\left|\phi_{i}(\xi, \eta)\right| \leqq M_{i} \leqq M$ in $R$;

(3) $\sum_{i, j}\left|\delta_{i j}-\partial \phi_{i} / \partial y_{j}\right|$ converges uniformly in $R$;

(4) the determinant $\left|\partial \phi_{i} / \partial y_{j}\right|$ is not zero at $(\alpha, \beta)$ with coordinates $a_{i}, b_{i}$

(5) $\phi_{i}(\xi, \eta)=0$ in $R$;

then unique solutions $y_{i}(\xi)$ of the system $\phi_{i}=0$ exist in a neighborhood of $(\alpha, \beta)$ and are completely continuous in a neighborhood of $\alpha .^{*}$

If

(6) $\partial \phi_{i} / \partial x_{k}$ are completely continuous in $R$;

(7) $\left|\partial \phi_{i} / \partial x_{k}\right| \leqq N_{k} \leqq N$ in $R$;

then $\partial y_{i} / \partial x_{k}$ exist in a neighborhood of $\alpha$, the equations

$$
\frac{\partial \phi_{i}}{\partial x_{k}}+\sum_{j} \frac{\partial \phi_{i}}{\partial y_{j}} \frac{\phi y_{j}}{\partial x_{k}}=0
$$

are satisfied, and $\left|\partial y_{i} / \partial x_{k}\right|<Q_{k}$ in the neighborhood.

DifFERENTIATION THEOREM. If $f(\eta)$ and $\partial f / \partial y_{i}$ are completely continuous in $T$;

$\left(8^{\prime}\right) \sum_{j}\left|\partial f / \partial y_{j}\right|$ converges uniformly in $T$;

$\left(9^{\prime}\right) y_{j}(v), d y_{j} / d v$ are continuous in $a \leqq v \leqq b$ and $\eta(v)$ lies in $T$; $\left(10^{\prime}\right) \sum_{j}\left|\left(\partial f / \partial u_{j}\right) \cdot\left(\partial y_{j} / \partial v\right)\right|$ converges uniformly in $a \leqq v \leqq b, y_{j}$ in $T$; then if $G(v)=f[\eta(v)]$,

$$
\frac{d G}{d v}=\sum_{i} \frac{\partial f}{\partial y_{i}} \frac{d y_{i}}{d v} .
$$

We now state the Lagrange multiplier theorem.

If $\phi_{i}(\xi, \eta)$-have the properties (1), . . (7); $f(\xi, \eta), \partial f / \partial x_{k}$, $\partial f / \partial y_{k}$ are completely continuous in $R$ and $f(\xi, \eta)$ has a maximum at $(\alpha, \beta)$ subject to the conditions $\phi_{i}(\xi, \eta)=0$ in $R$;

(8) $\sum_{i} \partial f / \partial y_{i}$ converges uniformly in $R$;

then there exist $\lambda_{i}$ such that $\sum_{i}\left|\lambda_{i}\right|$ converges and, at $(\alpha, \beta)$,

$$
\begin{aligned}
& \frac{\partial f}{\partial y_{k}}+\sum_{i} \lambda_{i} \frac{\partial \phi_{i}}{\partial y_{k}}=0, \\
& \frac{\partial f}{\partial x_{k}}+\sum_{i} \lambda_{i} \frac{\partial \phi_{i}}{\partial x_{k}}=0, \quad(k=1,2, \cdots) .
\end{aligned}
$$

* From Lemma 1 it follows that the assumption that $\sum_{k}\left|D_{k i}\right|$ is bounded in $i$, where $D_{k i}$ are the minors of the Jacobian of the system $\phi_{i}=0$, is redundant in Hart's Theorem XII. 
Proof. From conditions (3), (4), (8) and Lemma 2, it follows that (9) has a solution $\lambda_{i}$ with $\sum_{i}\left|\lambda_{i}\right|=\lambda$ at $(\alpha, \beta)$. The system $\phi_{i}=0$ has solutions $y_{i}(\xi)$. Condition ( $\left.8^{\prime}\right)$ follows from (8); $\left(9^{\prime}\right)$ from (1), $\cdots,(7)$; and $\left(10^{\prime}\right)$ from (6), (7), (8). Hence at the maximum

$$
\frac{\partial f}{\partial x_{k}}+\sum_{j} \frac{\partial f}{\partial y_{j}} \frac{\partial y_{j}}{\partial x_{k}}=0 .
$$

We note that

$$
\begin{aligned}
\sum_{i, j}\left|\lambda_{i} \frac{\partial \phi_{i}}{\partial y_{j}} \frac{\partial y_{j}}{\partial x_{k}}\right| & \leqq Q_{k} \sum_{i, j}\left|\lambda_{i} \frac{\partial \phi_{i}}{\partial y_{j}}\right| \\
& \leqq Q_{k}\left\{\sum_{i, j}\left|\lambda_{i}\right|\left|\frac{\partial \phi_{i}}{\partial y_{j}}-\delta_{i j}\right|+\sum_{i, j}\left|\lambda_{i} \delta_{i j}\right|\right\} \\
& \leqq Q_{k}\left\{\lambda \sum_{i, j}\left|\frac{\partial \phi_{i}}{\partial y_{j}}-\delta_{i j}\right|+\lambda\right\},
\end{aligned}
$$

and the convergence follows from (3). From (7) and (12) we have

$$
\begin{aligned}
\sum_{i} \lambda_{i} \frac{\partial \phi_{i}}{\partial x_{k}} & +\sum_{i} \lambda_{i} \sum_{j} \frac{\partial \phi_{i}}{\partial y_{j}} \frac{\partial y_{j}}{\partial x_{k}} \\
= & \sum_{i} \lambda_{i} \frac{\partial \phi_{i}}{\partial x_{k}}+\sum_{j} \frac{\partial y_{j}}{\partial x_{k}} \sum_{i} \lambda_{i} \frac{\partial \phi_{i}}{\partial y_{j}}=0 .
\end{aligned}
$$

Combining (11), (13), (9), we have

$$
\begin{aligned}
\frac{\partial f}{\partial x_{k}}+ & \sum_{j} \frac{\partial f}{\partial y_{j}} \frac{\partial y_{j}}{\partial x_{k}}+\sum_{i} \lambda_{i} \frac{\partial \phi_{i}}{\partial x_{k}}+\sum_{j} \frac{\partial y_{j}}{\partial x_{k}} \sum_{i} \lambda_{i} \frac{\partial \phi_{i}}{\partial y_{j}} \\
& =\frac{\partial f}{\partial x_{k}}+\sum_{i} \lambda_{i} \frac{\partial \phi_{i}}{\partial x_{k}}+\sum_{j} \frac{\partial y_{j}}{\partial x_{k}}\left[\frac{\partial f}{\partial y_{j}}+\sum_{i} \lambda_{i} \frac{\partial \phi_{i}}{\partial y_{j}}\right] \\
& =\frac{\partial f}{\partial x_{k}}+\sum_{i} \lambda_{i} \frac{\partial \phi_{i}}{\partial x_{k}}=0,
\end{aligned}
$$

which is (10), and the theorem is proved.

UNIVERSITY OF KENTUCKY 